\title{
To Study COVID 19 Infection among Healthcare Workers following Vaccination at Tertiary Care Centre
}

\author{
Anshudhar Maurya ${ }^{1}$, Rajendra Kumar Verma ${ }^{2, *}$, Pankaj Chaudhary ${ }^{3}$, Yogita Dwivedi ${ }^{4}$, Nirmala Singh ${ }^{5}$, \\ Nirbhay Dwivedi ${ }^{1}$
}

${ }^{1}$ Emergency Medical Officer, GMC, Azamgarh, Uttar Pradesh, INDIA.

${ }^{2}$ Associate Professor (Internal Medicine), GSVM, Medical College, Kanpur, Uttar Pradesh, INDIA.

${ }^{3}$ Assistant Professor (Pharmacology), GMC, Azamgarh, Uttar Pradesh, INDIA.

${ }^{4}$ Associate Professor (Anaesthesia), SNMC, Agra, Uttar Pradesh, INDIA.

${ }^{5}$ Senior Pediatrician, Consultant Pediatrician, Kanpur, Uttar Pradesh, INDIA.

\begin{abstract}
Introduction: More than 194 million cases have been confirmed, with more than 4.17 million confirmed deaths attributed to COVID-19 till July 2021, making it one of the deadliest pandemics in history. Since the advent of the disease the only way to contain the spread and prevent infection besides personal precaution was invention of vaccine. Since healthcare workers were at highest risk of exposure to infection, we planned to study whether there were chances of getting infected after vaccination for Covid 19 and if so then when and what was the severity of infection. Materials and Methods: A randomized, cross-sectional online survey was performed from 26 May to 10 June among health care workers in a tertiary care hospital. A self-administered online survey (created with Google Forms) that was circulated via social media platforms. Questions were formatted in binary fashion to the extent possible, with descriptive features added to the section on symptom profile. All quantitative variables are presented as mean and standard deviation, and all qualitative variables in frequency and percentages. All the data were entered in Microsoft excel. Result: Out of 800 HCW's 520 of them responded to the survey. Prevalence rate was 9.25\%. Majority of the HCW had developed symptoms from two week to two months. Disease severity was mild in 46 cases (92\%), moderate in 2 cases (4\%) and severe in 2 cases (4\%). Majority of HCW's with Covid symptoms post vaccination were Interns (46\%), followed by nurses (20\%) Faculty $(14 \%)$ and others. Fever was the most common symptom followed by sore throat, body ache, malaise and cough. OPD basis treatment and home isolation was advised in 37 cases $(84 \%)$ while 13 cases (26\%) required hospitalization. No Mortality was seen. Conclusion: Higher prevalence of disease coincides with the higher exposure rate (surge in the Covid 19 cases). Adequate and timely vaccination could play a major role in fighting with the deadliest pandemic by curtailing transmission rate, severity of the disease and mortality.
\end{abstract}

Key words: Post vaccination, Healthcare Workers, COVID-19 Infection, Prevalence, Disease Severity.
Article Information

Received: 01-08-2021

Revised : 10-10-2021

Accepted: 14-12-2021

Correspondence

Dr. Rajendra Kumar Verma

Associate Professor, Medicine, GSVM Medical College, Kanpur-208002, Uttar Pradesh, INDIA.

E-mail: drrkverma.research@gmail.com

\begin{tabular}{|l|c|}
\hline \multicolumn{2}{|c|}{ Access this article online } \\
\hline Website: & Quick Response code \\
\cline { 2 - 2 } www.jcramonline.com & \\
\hline DOI: & \\
10.5530/jcram.1.2.12 & \\
\hline
\end{tabular}

\section{INTRODUCTION}

An ongoing global pandemic of coronavirus disease 2019 (COVID-19) caused by severe acute respiratory syndrome coronavirus 2 (SARS-CoV-2) first hit the headlines in December 2019 from Wuhan, China. ${ }^{1}$ Since then it had spread all over the world in short span of time. It was declared pandemic by WHO 
(World Health Organization) on March 10, 2020.2 As of 27 July 2021, more than 194 million cases have been confirmed, with more than 4.17 million confirmed deaths attributed to COVID-19, making it one of the deadliest pandemics in history.

Several countries including India have seen a two-wave pattern of COVID cases. Since the advent of the disease the only way to contain the spread and prevent infection besides personal precaution was invention of vaccine. Various forms of vaccines such as Covishield vaccine (Viral Vector based Technology), Covaxin vaccine (wholeVirion Inactivated Corona Virus), ${ }^{3}$ inactivated (PiCoVacc), live attenuated (CDX-CoV), viral vector (Sputnik V), protein subunit (Novavax) and nucleic acid (DNA (Zydus Cadila), RNA (Pfizer, Moderna) are been developed. ${ }^{4}$

In India vaccination campaign for COVID-19 was started on January 16, 2021 using two vaccines Covishield and Covaxin. Vaccination in our center was started from February 16, 2021. Initial doses were given to health care workers, front line workers and people with more than 45 years of age with morbidities (like diabetes, coronary artery disease etc.). From May 1st onwards vaccination is open to all individuals $(>18 \mathrm{y}$ of age). Since healthcare workers were at highest risk of exposure to infection, we planned to study whether there were chances of getting infected after vaccination for Covid 19 and if so then when and what was the severity of infection.

\section{Aim}

- To study the prevalence of COVID-19 infection in post vaccinated HCWs

- To study post vaccination disease severity in HCWs.

\section{MATERIALS AND METHODS}

A randomized, cross-sectional online survey was performed from 26 may to 10 June among health care workers in a tertiary care hospital with 300 bedded Covid health care facility. They were asked to take part in a self-administered online survey (created with Google Forms) that was circulated via social media platforms. Potential participants were directed to a page that included a thorough description of the study's purpose before being asked to agree to a mandatory electronic informed consent form that included statements about voluntary participation and anonymity. The study's survey tool (Supplementary Material) was developed after an extensive literature search that included Medline/PubMed, Google Scholar, and other databases. The survey tool was written in English, and it was validated by a panel of experts who provided feedback on the different items of the survey, which were then updated based on their suggestions.

Questions were formatted in binary fashion to the extent possible, with descriptive features added to the section on symptom profile. Provision was provided to add other outcomes or descriptions that further qualified the experience. Descriptive Statistics were used to assess the baseline characteristics of the data. All quantitative variables are presented as mean and standard deviation, and all qualitative variables in frequency and percentages. All the data were entered in Microsoft excel.

\section{RESULTS}

Out of 800 persons included in the survey, $520 \mathrm{HCW}$ 's responded to the survey over a two-week period from 25 may to 10 June 2021. Among those who responded to the survey 9.25\% HCW's (50) were positive to RT-PCR covid 19 after vaccination where as $90.75 \%$ of HCW's were not positive for covid 19 despite of exposure to covid positive patients owing to their active duties in hospital. (Figure 1)

Out of 50 Positive patients 52\% were females and $48 \%$ males. (Figure 2) Out of 26 positive females 53.84\% were in the age group of $20-30$ and similarly out of 24 positive males patients $75 \%$ were of age $20-30$ years followed by the age group of $31-40$ years. (Table 1 , Figure 2, Figure 3).

Majority of HCW's with covid symptoms post vaccination were Interns (46\%), followed by nurses (20\%) Faculty (14\%) and others. Among males 54.16\% were interns, $16.66 \%$ residents, $12.5 \%$ faculty, $8.32 \%$ graduate students and $4.16 \%$ paramedical and others each. While among females $38.46 \%$ were interns, $15.38 \%$ faculty and $7.69 \%$ others. All the nurses were females. (Table 2, Figure 4)

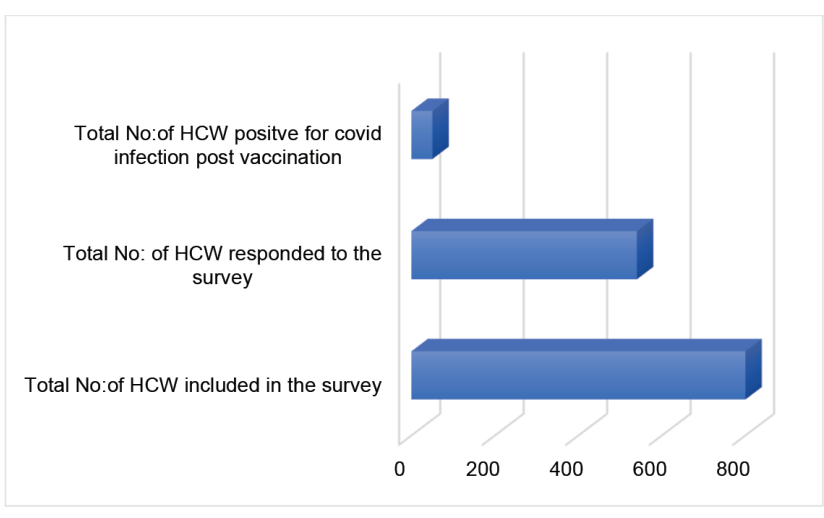

Figure 1: Study Participants Statistics. 
Table 1: HCWs Characteristics Stratified by Type of vaccine and Vaccination-to-Diagnosis Timing.

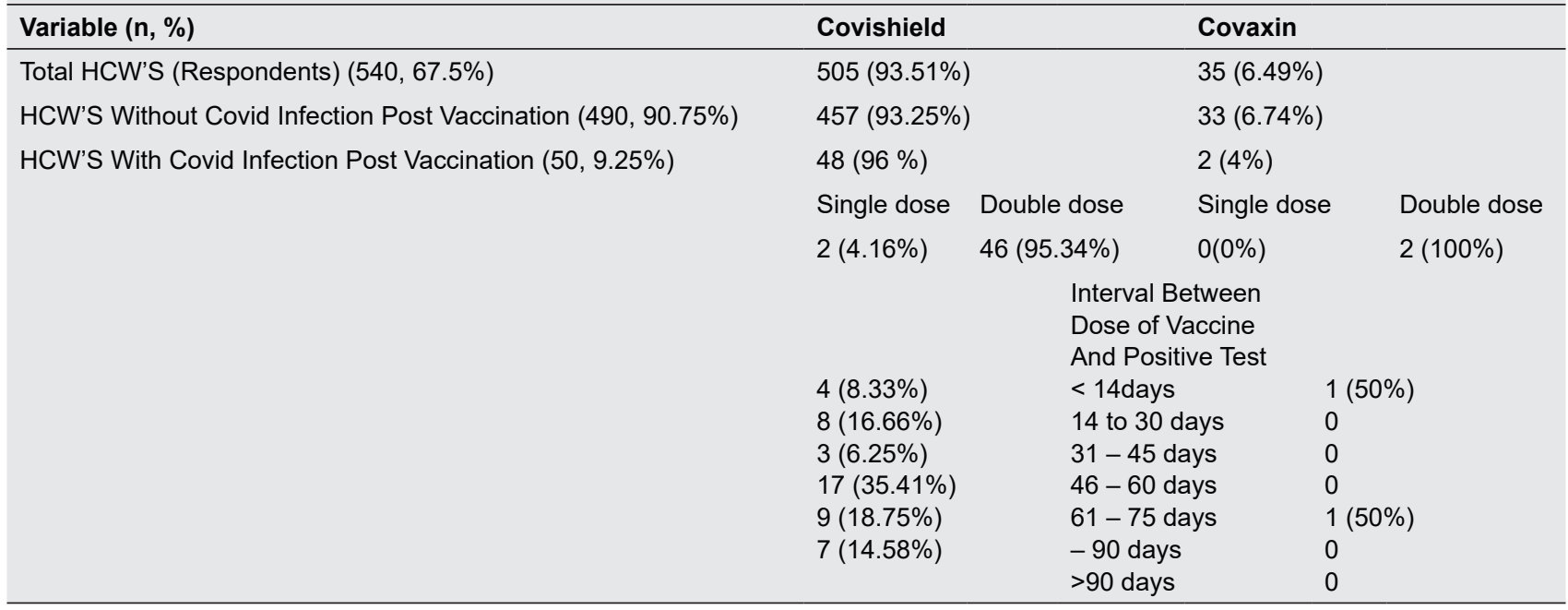

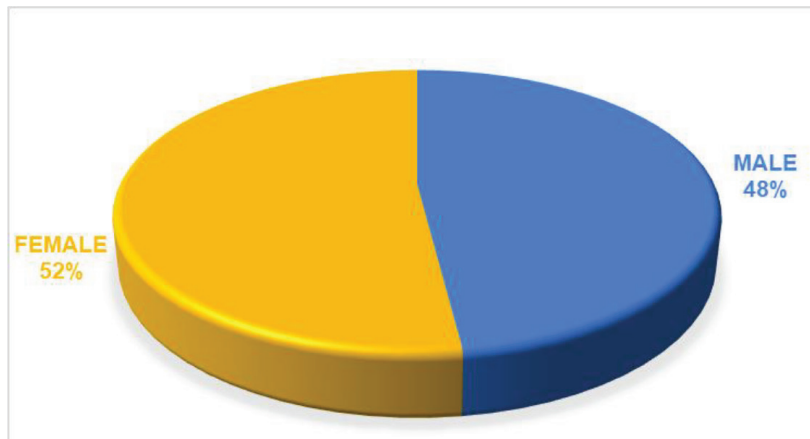

Figure 2: Distribution of Study Subjects Based of Sex.

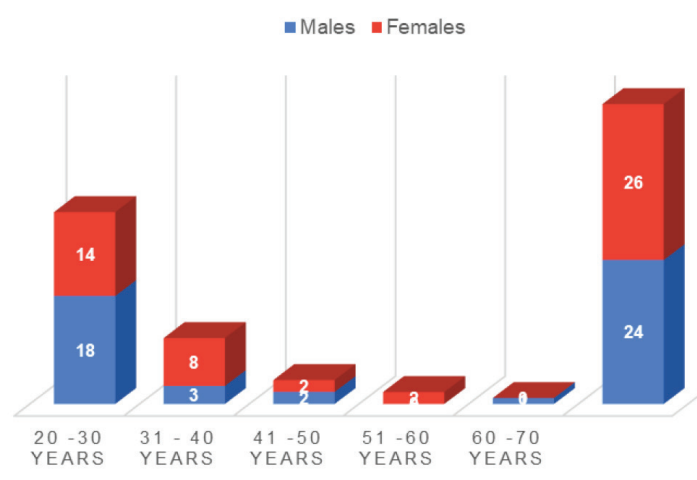

Figure 3: Age Based Distribution of Study Participants in Our Study.

Among the respondents, 505 (93.51\%) had received Covishield (Astra-Oxford vaccine manufactured by Serum Institute, India), 35 (6.74\%) received Covaxin (Bharat Biotech, India). 96\% (48) of HCW's with post vaccination covid 19 infection had received Covishield

\begin{tabular}{|c|c|c|}
\hline $\begin{array}{l}\text { Variable }(n, \%) \\
\text { Total covid positive } \\
\text { HCW's post } \\
\text { vaccination (50) }\end{array}$ & $\begin{array}{l}\text { Males } \\
(24,48 \%)\end{array}$ & $\begin{array}{l}\text { Females } \\
(26,52 \%)\end{array}$ \\
\hline \multicolumn{3}{|l|}{$\begin{array}{l}\text { Categories of Working } \\
\text { Profile }\end{array}$} \\
\hline Faculty $(7,14 \%)$ & $3(12.5 \%)$ & $4(15.38 \%)$ \\
\hline Residents $(4,8 \%)$ & $4(16.66 \%)$ & $0(0 \%)$ \\
\hline Interns $(23,46 \%)$ & $13(54.16 \%)$ & $10(38.46 \%)$ \\
\hline Nurses $(10,20 \%)$ & $0(0 \%)$ & $10(38.46 \%)$ \\
\hline Paramedical $(1,2 \%)$ & $1(4.16 \%)$ & $0(0 \%)$ \\
\hline MBBS students $(2,4 \%)$ & $2(8.32 \%)$ & $0(0 \%)$ \\
\hline Others $(3,6 \%)$ & $1(4.16 \%)$ & $2(7.69 \%)$ \\
\hline \multicolumn{3}{|l|}{ Age Categories } \\
\hline $20-30$ years $(32,64 \%)$ & $18(75 \%)$ & $14(53.84 \%)$ \\
\hline $31-40$ years $(11,22 \%)$ & $3(12.5 \%)$ & $8(30.76 \%)$ \\
\hline $41-50$ years $(4,8 \%)$ & $2(8.33 \%)$ & $2(7.69 \%)$ \\
\hline $51-60$ years $(2,4 \%)$ & $0(0 \%)$ & $2(7.69 \%)$ \\
\hline $60-70$ years $(1,2 \%)$ & $1(4.16 \%)$ & $0(0 \%)$ \\
\hline
\end{tabular}

of which 95.34\% (46) developed symptoms after double dose and only $4.16 \%$ (2) after first dose. While only $4 \%$ received Covaxin and all of them developed were symptomatic after double dose of vaccination. Majority of HCW,s 35.41\% developed COVID-19 infection between $1 \frac{1 / 2}{2}$ months to two months followed by $18.75 \%$ of them between 2 months to $2^{1 / 2}$ months later to the last dose of vaccination, whereas among Covaxin group 50\% developed symptoms within two weeks and rest 50\% between $2-2 \frac{1}{2}$ months. Categories. (Table 1 ). Symptomatic cases were 46 (92\%) and asymptomatic were $4(8 \%)$. Fever was the most common symptom followed by sore throat, body ache, malaise and cough. 


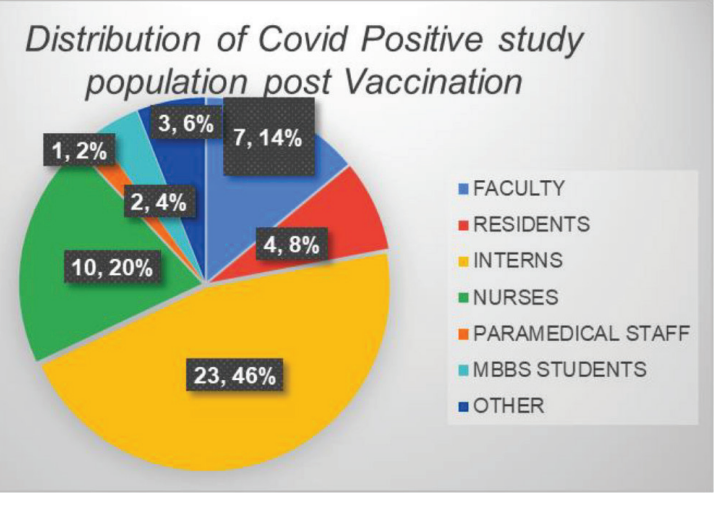

Figure 4: Distribution of covid 19 positive study population post vaccination.

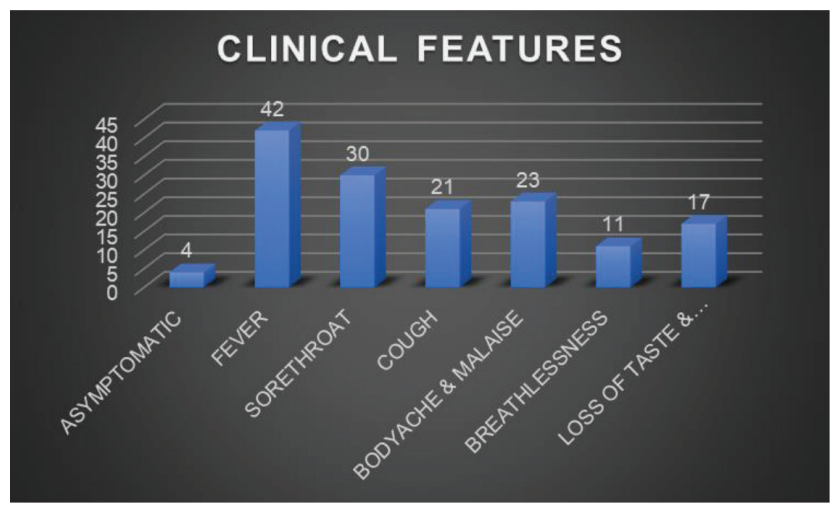

Figure 5: Post vaccination COVID-19 disease presentation.

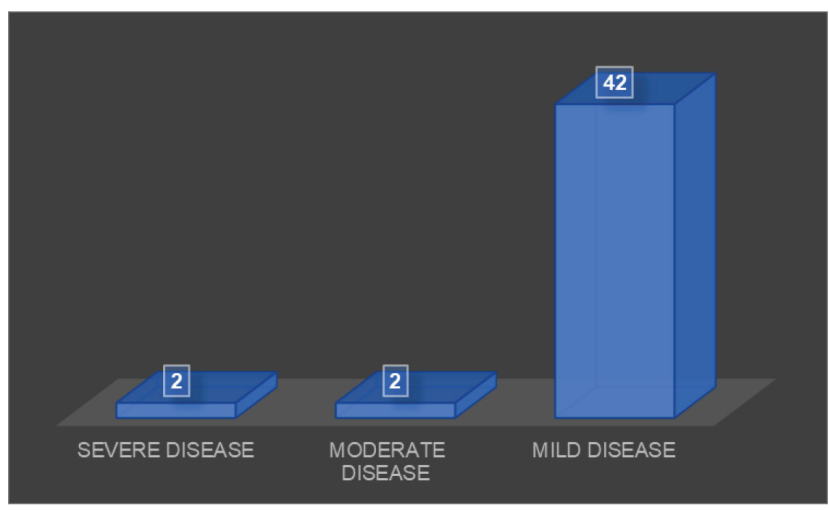

Figure 6: Post vaccination COVID-19 disease severity status.

Breathlessness was present in only $11(22 \%)$ out which only one patient $(2 \%)$ developed severe respiratory distress and was treated in ICU followed by complete recovery. (Figure 5)

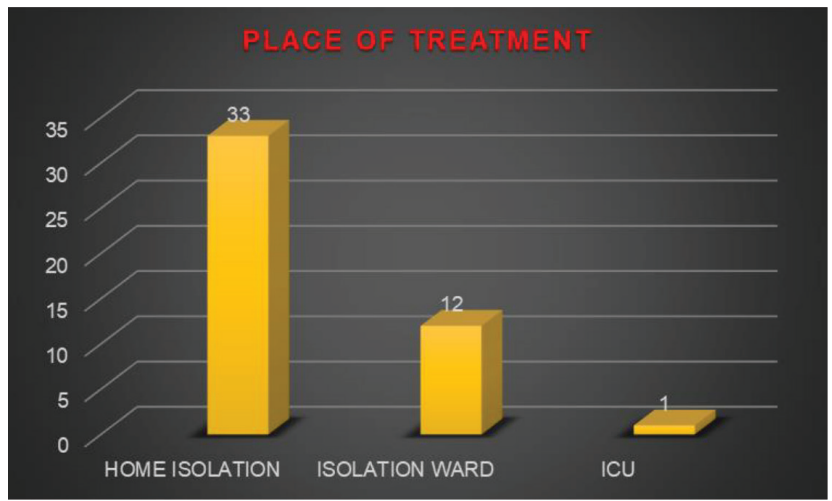

Figure 7: Place (Home isolation, Isolation ward, ICU) statistics.

OPD basis treatment and home isolation was advised in 37 cases $(84 \%)$ while 13 cases $(26 \%)$ required hospitalization. All severe (4\%), moderate cases (4\%) and few mild cases were admitted. Disease severity was mild in 46 cases (92\%), moderate in 2 cases (4\%) and severe in 2 cases (4\%). (Figure 6). Out of 13 hospitalized cases one case required ICU admission while rest of them were managed in Isolation ward. No Mortality was seen. (Figure 7).

\section{DISCUSSION}

SARS-CoV-2 is different from other known viruses due to multiple mutations on the sites of nonstructural proteins (NSP) 2 and 3, and the varying nature of virulence between different persons. ${ }^{4}$ Multiple waves of transmission during infectious disease epidemics represent a major public health challenge. ${ }^{5}$ Several studies emphasized that newer SARS-CoV-2 variants have increased infectivity, modest decrease in neutralizing activity, and may impact vaccine effectiveness via escape from vaccine-induced immunity, specifically by mutations in the spike protein. ${ }^{6}$ Efficacious vaccine is an ultimate conclusive solution to combat the pandemic.

The Subject Expert Committee of Central Drugs Standard Control Organization (CDSCO) made recommendations in respect of proposal for Restricted Emergency Approval of COVID-19 virus vaccine of $\mathrm{M} / \mathrm{s}$ Serum Institute of India (Covishield) and M/s Bharat Biotech (Covaxin) as well as Phase III clinical trial of $\mathrm{M} / \mathrm{s}$ Cadila Healthcare Ltd in a meeting held on 1st and 2nd January, 2021. 
Covishield is a Recombinant Chimpanzee Adenovirus vector Vaccine encoding the SARS-CoV-2 Spike (S) glycoprotein with technology transfer from AstraZeneca/ Oxford University. The firm (Serum Institute of Pune) submitted safety, immunogenicity and efficacy data generated on 23,745 participants aged $\geq 18$ years or older from overseas clinical studies. The overall vaccine efficacy was found to be $70.42 \%$.

Bharat Biotech has developed a Whole Virion Inactivated Corona Virus Vaccine (Covaxin) in collaboration with ICMR and NIV (Pune), from where they received the virus seed strains. BBV152 contains a whole virion inactivated SARS-CoV-2 vaccine, which is produced in Vero cells. It is stable at 2 to $8^{\circ} \mathrm{C}$ (refrigerated). Interim Phase 3 Results showed 81\% vaccine Efficacy.

In our study all the respondents after vaccination worked in hospital environment during the second peak (April to mid of May) of covid 19 disease. They were all at high risk for exposure to covid 19 infection. High prevalence rate $(9.25 \%)$ in our study was consistent with the findings of Patil et al. (Prevalence rate 10.1\%). ${ }^{7}$ Whereas several studies like that of Bouton et al. study had prevalence of $1.3 \%(96 / 7,109)$ infected HCWs who received at least one dose and $0.3 \%(17 / 5,913) \mathrm{HCW}$ given both doses. ${ }^{8}$ Amit $S$ et al. study showed $0.54 \%$ infection prevalence in vaccinated HCWs. ${ }^{9}$ Thompson et al. study had prevalence of $5.2 \%(205 / 3,950) .{ }^{10}$ Reason of this difference are many. Our study includes $\mathrm{HCW}$ as compared to other studies which include general population. Also most of the HCW in this study are frontline HCWs working in COVID ICU, Wards, Lab Areas dedicated to covid 19 work. The time period of study (March, April and may) coincided with the second surge of Pandemic in India.

Most of the HCWs worked together and stayed in near buildings, were in touch with local community so interpersonal and community transmission was also the possibility. Asymptomatic cases were detected because of Contract tracing. Also HCWs had an easy accessibility for testing as compared to General public. Among the covid positive HCW, Majority were of younger age in both the sex were as the older group had a lower positive rate. In our study interns, Nurses and residents constituted more than $70 \%$ of the Positive HCW. Possible reasons for Difference might be because of Experience, Knowledge, and Precautionary Measures. In our center Vaccination was done to majority in February, March that is before the surge in second wave of covid 19. In our study Majority (58.32\%) of cases occurred between two weeks to two months after vaccination. This period of exposure matches with the peak period (April - May) of surge in cases of covid 19 patients in the hospital which clearly indicates increase in exposure rates for contacting covid 19 virus. Prevalence of infection in HCW could have been higher in absence of vaccination. Except for two health care workers severity of the disease in rest of them was predominantly mild to moderate which were treated easily either in home of hospital isolation wards. Both the severely diseased HCW's also recovered with leaving behind mortality rate to zero. Had it been no vaccination then prevalence rate, mortality rate could have been more. An efficacious COVID-19 vaccine can reduce the likelihood of infection of an individual, severity of disease and degree of transmission within a population. ${ }^{3}$

\section{Limitations}

Sample size was smaller in our study. Online survey is more likely to be taken by those with an interest in the topic.

\section{CONCLUSION}

HCWs and frontline workers being at highest risk of exposure were vaccinated first. Higher prevalence of disease coincides with the higher exposure rate (surge in the COVID-19 cases). Adequate and timely vaccination could play a major role in fighting with the deadliest pandemic by curtailing transmission rate, severity of the disease and mortality.

\section{CONFLICT OF INTEREST}

The authors declare no conflict of interest.

\section{REFERENCES}

1. Page J, Hinshaw D, McKay B. In Hunt for Covid-19 Origin, Patient Zero Points to second Wuhan Market - the man with the first confirmed infection of the new coronavirus told the WHO team that his parents had shopped there. The Wall Street Journal. [retrieved Feb 27 2021]; February 262021.

2. Adhanom T. "WHO Director-General's remarks at the media briefing on. nCoV on 11 February 2020. World Health Organization (World Health Organization); 2019-.

3. FAQs on COVID 19 vaccine for Healthcare providers and. Available from: https://www.mohfw.gov.in [cited 28/9/2021] ) FAQsfor HCWs \&and FLWs.

4. Sampath Kumar NS, Chintagunta AD, Jeevan Kumar SP, Roy S, Kumar M. Immunotherapeutics for Covid-19 and post vaccination surveillance. 3 Biotech. 2020;10(12):527. doi: 10.1007/s13205-020-02522-9, PMID 33200061. 
5. Kaxiras E, Neofotistos G. Multiple epidemic wave model of the COVID-19 pandemic: modeling study. J Med Internet Res. 2020;22(7):e20912. doi: 10.2196/20912, PMID 32692690.

6. Abdool Karim SS, de Oliveira T. New SARS-CoV-2 Variants - clinical, public health, and vaccine implications. NEngl J Med. 2021 March 24;384(19):1866-8. doi: 10.1056/NEJMc2100362, PMID 33761203.

7. Patil $\mathrm{Y}$, Kesari M, Agrawal S, Dholpure M, Reheman $\mathrm{H}$. Post vaccination covid infection in health care workers at a tertiary care centre: a retrospective cohort study. Int J Contemp Med Res. 2021;8(5):E1-5. doi: 10.21276/ ijcmr.2021.8.5.2.

8. Bouton TC, Lodi S, Turcinovic J, Weber SE, Quinn E. COVID-19 vaccine impact on rates of SARS-CoV-2 cases and post vaccination strain sequences among healthcare workers at an urban academic medica center: a prospective cohort study. medRxiv [preprint]. 2021.03.30.21254655.

9. Amit S, Beni SA, Biber A, Grinberg A, Leshem E, Regev-Yochay G. Postvaccination COVID-19 among healthcare workers, Israel. Emerg Infect Dis. 2021;27(4):1220-2. doi: 10.3201/eid2704.210016, PMID 33522478.

10. Thompson MG, Burgess JL, Naleway AL, Tyner HL, Yoon SK, Meece J, et al. Interim Estimates of Vaccine Effectiveness of BNT162b2 and mRNA1273 COVID-19 Vaccines in Preventing SARS-CoV-2 Infection Among Health Care Personnel, First Responders, and Other Essential and Frontline Workers - Eight US Locations, December 2020-March 2021. MMWR Morb Mortal Wkly Rep. 2021;70(13):495-500. doi: 10.15585/mmwr.mm7013e3, PMID 33793460.

Cite this Article : Maurya A, Verma RK, Chaudhary P, Dwivedi Y, Singh N, Dwivedi N. To Study COVID-19 Infection among Healthcare Workers Following Vaccination at Tertiary Care Centre. J Clinical Res and Appl Med. 2021;1(2):48-53. 